Research Paper

\title{
Antibiotic resistance profiles of deep surgical site infections in hip hemiarthroplasty; comparing low dose single antibiotic versus high dose dual antibiotic impregnated cement
}

\author{
Ben Tyas $^{1 凶}$, Martin Marsh² , Tamsin Oswald 3 , Ramsay Refaie ${ }^{4}$, Catherine Molyneux ${ }^{5}$, Mike Reed ${ }^{6}$ \\ 1. Foundation Doctor, University Hospital North Durham, North Road, Durham, DH1 5TW, United Kingdom \\ 2. Clinical Research Fellow, Northumbria Specialist Emergency Healthcare Centre, Northumbrian Road, Cramlington, NE23 6NZ, United Kingdom \\ 3. Consultant Microbiologist, Northumbria Specialist Emergency Healthcare Centre, Northumbrian Road, Cramlington, NE23 6NZ, United Kingdom \\ 4. Clinical Research Fellow, Northumbria Specialist Emergency Healthcare Centre, Northumbrian Road, Cramlington, NE23 6NZ, United Kingdom \\ 5. Consultant Microbiologist, Royal Victoria Infirmary, Queen Victoria Road, NE1 4LP, United Kingdom \\ 6. Consultant Trauma and Orthopaedic Surgeon, Northumbria Specialist Emergency Healthcare Centre, Northumbrian Road, Cramlington, NE23 6NZ, \\ United Kingdom \\ $\bowtie$ Corresponding author: Telephone: +447794225785; Email: b.tyas@outlook.com
}

(c) Ivyspring International Publisher. This is an open access article distributed under the terms of the Creative Commons Attribution (CC BY-NC) license (https://creativecommons.org/licenses/by-nc/4.0/). See http://ivyspring.com/terms for full terms and conditions.

Received: 2017.08.01; Accepted: 2018.02.04; Published: 2018.06.11

\begin{abstract}
Objectives: The incidence of fractured neck of femur (FNOF) is increasing yearly. Many of these patients undergo hip hemiarthroplasty. High dose dual-antibiotic cement (HDDAC) has been shown to reduce rates of deep surgical site infection (SSI) when compared to the current standard low dose single-antibiotic cement (LDSAC) in a quasi-randomised controlled trial. Some concerns exist regarding the use of HDDAC and the development of antibiotic resistance. We reviewed cases of infection in LDSAC and HDDAC bone cement with regard to causative organism and resistance profile.

Methods: A retrospective analysis was undertaken of all hemiarthroplasties within our trust from April 2008 to December 2014. We identified all patients in this time period who acquired a deep SSI. The infecting organisms and susceptibility patterns were collated for each cement.

Results: We identified 1941 hemiarthroplasties. There were 38 deep surgical site infections representing an infection rate of $3.4 \%$ in LDSAC patients and $1.2 \%$ in HDDAC patients. The majority of infections were polymicrobial. Staphylococcus epidermidis was the most commonly isolated organism. It accounted for a larger proportion of HDDAC than LDSAC infections $(p<0.05)$. Infection with Corynebacterium species and $S$. aureus, including MRSA, was eradicated completely with the use of HDDAC. There was no significant change in the proportion of Gram negative and Gram positive infections between the two cements. In Gram positive organisms, there was no significant change in resistance to most antibiotics. Although fewer resistant infections overall, there were significant increases in the proportion of resistance to ciprofloxacin and clindamycin with HDDAC. We observed no resistance to daptomycin or linezolid in either cement and levels of resistance remained low to rifampicin and teicoplanin. In Gram negative organisms, no significant change in resistance was observed.

Conclusions: We observed a significantly lower infection rate with the use of HDDAC compared to LDSAC. Such was this reduced infection rate that there was a trend to a lower rate of resistance with the use of HDDAC. However, there were increases in the proportion of resistant cases, most notably to clindamycin and ciprofloxacin in Gram positive organisms, possibly reflecting the higher number of $S$. epidermidis in the HDDAC group. Whilst the differences in our study were not found to be statistically significant, it is reassuring for teams using HDDAC to prevent SSI in hip hemiarthroplasty.
\end{abstract}




\section{Introduction}

The incidence of fractured neck of femur (FNOF) is increasing. It is estimated that worldwide there will be approximately 2.6 million cases by 2025; more than double the number in 1990.1 According to the National Hip Fracture Database there were 65,000 cases of FNOF in 2015 and $83.6 \%$ of these patients underwent cemented hip arthroplasty ${ }^{2}$. This subset of patients has high rates of peri-operative complications. ${ }^{3}$ Surgical site infection (SSI) is one of the most devastating complications and is associated with increased length of stay and increased mortality. ${ }^{4}$ Infection rates between $1.3 \%$ and $7 \%$ have been reported for hemiarthroplasty, with the UK rate of $4.06 \% .^{5}$ A quasi-randomised controlled trial conducted by Sprowson et al. recently demonstrated that high dose dual-antibiotic cement (HDDAC) (Copal G+C, Heraeus Medical, UK) reduces rates of deep SSI when compared to the standard low dose single-antibiotic cement (LDSAC) (Palacos R+G, Heraeus Medical, UK). ${ }^{6}$ Palacos R+G contains 0.5 grams of gentamicin whilst Copal $\mathrm{G}+\mathrm{C}$ contains 1 gram of gentamicin and 1 gram of clindamycin per mix. The addition of clindamycin to gentamicin-loaded bone cement is known to work synergistically to yield higher antibiotic release in vivo as well as providing a wider antibiotic coverage. ${ }^{7}$ It has also been shown to be more effective in preventing biofilm formation. ${ }^{8}$ However, concerns have been raised regarding antibiotic resistance with the use of antibiotic-loaded bone cement. ${ }^{9,10} \mathrm{We}$ reviewed the causative organisms and resistance profiles of all cases of infection in a large series of hemiarthroplasties where either LDSAC or HDDAC was used.

\section{Patients included in this study}

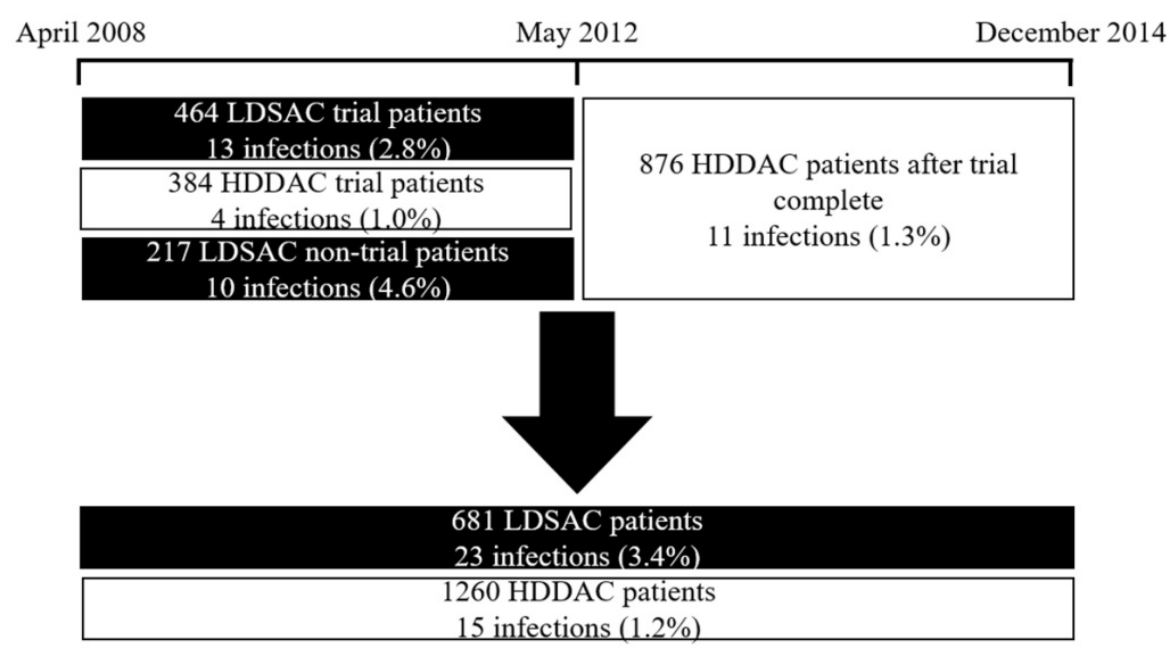

Figure 1. Patients included in our study with associated infection rate of each group

\section{Materials and methods}

A retrospective analysis was undertaken of all hemiarthroplasties between April 2008 and December 2014 at Wansbeck General Hospital and North Tyneside General Hospital, part of Northumbria Healthcare NHS Foundation Trust in the UK. We identified all patients in this period who acquired a deep SSI from the trust SSI surveillance database. Surveillance is performed routinely within the trust by dedicated orthopaedic SSI surveillance nurses. This period includes patients recruited into the quasi-randomised controlled trial. ${ }^{6}$ This trial ran from April 2008 to May 2012 and randomised patients to receive LDSAC or HDDAC for their hip hemiarthroplasty. Following completion of the trial the trust switched to HDDAC as the standard of care for hip hemiarthroplasty. A detailed breakdown of patients included in this study is shown in Figure 1. All these patients received systemic prophylactic antibiotics according to our trust protocol for hip hemiarthroplasty (teicoplanin and gentamicin, or aztreonam instead of gentamicin in the context of renal impairment). We collated clinical results for infective organisms and susceptibility patterns for infections in each cement. In our trust, orthopaedic tissues and fluids are cultured for five days direct, then the enrichment broth is sub-cultured out onto plates and this is then read after 48 hours. Fluid aspirates are inoculated into blood culture bottles and then plated at seven days. The plates are then read after 72 hours (i.e. ten days in total). These methods are consistent with UK standards for microbiology investigations. ${ }^{11}$ Sensitivity was determined using automated sensitivity testing on the VITEK $® 2$ system. Breakpoints and zone sizes established by CLSI or BSAC were used depending on the organism and antibiotic tested. The VITEK®2 system in our trust changed from using BSAC to CLSI breakpoints in March 2012. Manual CLSI susceptibility testing was introduced in June 2012 and was used in a few cases, such as for unusual isolates that failed on VITEK $® 2$, and where there were no VITEK® cards available for that particular organism.

\section{Results}

We identified 1941 hemiarthroplasties. 681 of these patients received LDSAC and 1260 received HDDAC. 


\section{Infection rate}

There were 38 deep surgical site infections; 23 in LDSAC and 15 in HDDAC, representing an infection rate of $3.4 \%$ in LDSAC patients and 1.2\% in HDDAC patients. The deep infection rate in HDDAC patients outside the trial was $1.3 \%$. These figures are comparable to the quasi-randomised controlled trial previously conducted. ${ }^{6}$

\section{Causative organisms}

Two patients in the LDSAC group were classified with deep infection according to the Public Health England definition, however, these patients were too unwell to undergo any further investigation of their infection and later died. We also excluded a further two deep LDSAC infections for which only superficial swab samples were obtained. This left 19 LDSAC and 15 HDDAC infections for further analyses. Of these, the majority of infections were polymicrobial (Table 1). In polymicrobial infections, $S$. epidermidis was the most commonly isolated organism, found in 4 of the LDSAC polymicrobial infections and 5 of the HDDAC polymicrobial infections. It also accounted for several other infections where it was the only isolated organism (Table 1). It accounted for a larger proportion of HDDAC than LDSAC infections $(\mathrm{p}<0.05)$ (Table 1). Infection with Corynebacterium species and Staphylococcus aureus, including MRSA, was eradicated completely with the use of HDDAC (Table 1 ). There was no difference in the proportion of Gram negative $(p>0.05)$ and Gram positive $(p>0.05)$ infections between the two cements (Table 1).

\section{Resistance profile}

Within the Gram positive organisms, there was no significant change in resistance to most antibiotics with the use of HDDAC compared to LDSAC (Table 2 , Figure 2). The exceptions being significant increases in resistance to ciprofloxacin and clindamycin. All organisms isolated on HDDAC that tested against erythromycin were found to be resistant, though this was not found to be a statistically significant difference to LDSAC $(p=0.064)$. Little or no resistance was observed to daptomycin, linezolid, rifampicin, teicoplanin and vancomycin in both groups.

Table 1. Breakdown of causative organisms in isolated infections

\begin{tabular}{|c|c|c|c|}
\hline & Cement & & \\
\hline & LDSAC & HDDAC & Chi Squared \\
\hline Total Number of Infections & $23(3.4 \%)$ & $15(1.2 \%)$ & $0.003^{* *}$ \\
\hline Excluded & 4 & 0 & \\
\hline Gram +ve infections & $10(43.48 \%)$ & $9(60.00 \%)$ & 0.332 \\
\hline Gram -ve infections & $4(17.39 \%)$ & $3(20.00 \%)$ & 0.809 \\
\hline Mixed gram status & $5(21.74 \%)$ & $3(20.00 \%)$ & 0.885 \\
\hline Monomicrobial & $9(39.13 \%)$ & $7(46.67 \%)$ & 0.641 \\
\hline Polymicrobial & $10(43.48 \%)$ & $8(53.33 \%)$ & 0.563 \\
\hline Monomicrobial (gram +ve) & $7(30.43 \%)$ & $5(33.33 \%)$ & 0.839 \\
\hline Staphylococcus epidermidis & $2(8.70 \%)$ & $5(33.33 \%)$ & $0.001^{* *}$ \\
\hline Staphylococcus aureus & $3(13.04 \%)$ & $0(0.00 \%)$ & 0.162 \\
\hline MRSA & $1(4.35 \%)$ & $0(0.00 \%)$ & 0.419 \\
\hline Staphylococcus haemolyticus & $1(4.35 \%)$ & $0(0.00 \%)$ & 0.419 \\
\hline Monomicrobial (gram -ve) & $2(8.70 \%)$ & $2(13.33 \%)$ & 0.542 \\
\hline Proteus mirabilis & 2 & & \\
\hline Klebsiella pneumoniae & & 1 & \\
\hline Pseudomonas aeroginosa & & 1 & \\
\hline Polymicrobial (gram +ve) & $3(13.04 \%)$ & $4(26.67 \%)$ & 0.144 \\
\hline Corynebacterium, S. epidermidis & 1 & & \\
\hline S. epidermidis, S. haemolyticus, Gemella sanguinis & 1 & & \\
\hline MRSA, Enterococcus faecium & 1 & & \\
\hline S. epidermidis, Enterococcus faecalis & & 2 & \\
\hline S. aureus, S. haemolyticus & & 1 & \\
\hline S. epidermidis, S. haemolyticus, Enterococcus faecium & & 1 & \\
\hline Polymicrobial (gram -ve) & $2(8.70 \%)$ & $1(6.67 \%)$ & 0.790 \\
\hline Proteus mirabilis, Klebsiella pneumoniae & 1 & & \\
\hline Klebsiella oxytoca, E. coli, Morganella morganii & 1 & & \\
\hline Pseudomonas aeroginosa, Enterobacter clocae & & 1 & \\
\hline Polymicrobial (mixed gram status) & $5(21.74 \%)$ & $3(20.00 \%)$ & 0.885 \\
\hline Enterococcus faecalis, S. epidermidis, Pseudomonas aeroginosa, Klebsiella pneumoniae & 1 & & \\
\hline MRSA, E. coli & 1 & & \\
\hline S. epidermidis, Enterococcus faecalis, E. coli & 1 & & \\
\hline Serratia marcesens, Enterococcus faecium & 1 & & \\
\hline E. coli, Enterococcus faecium & 1 & & \\
\hline Proteus mirabilis, Enterococcus avium, S. haemolyticus & & 1 & \\
\hline S. epidermidis, Proteus mirabilis & & 1 & \\
\hline Enterococcus faecalis, Proteus mirabilis, Serratia marcesens & & 1 & \\
\hline
\end{tabular}




\section{Percentage of Resistant Gram positive Organisms}

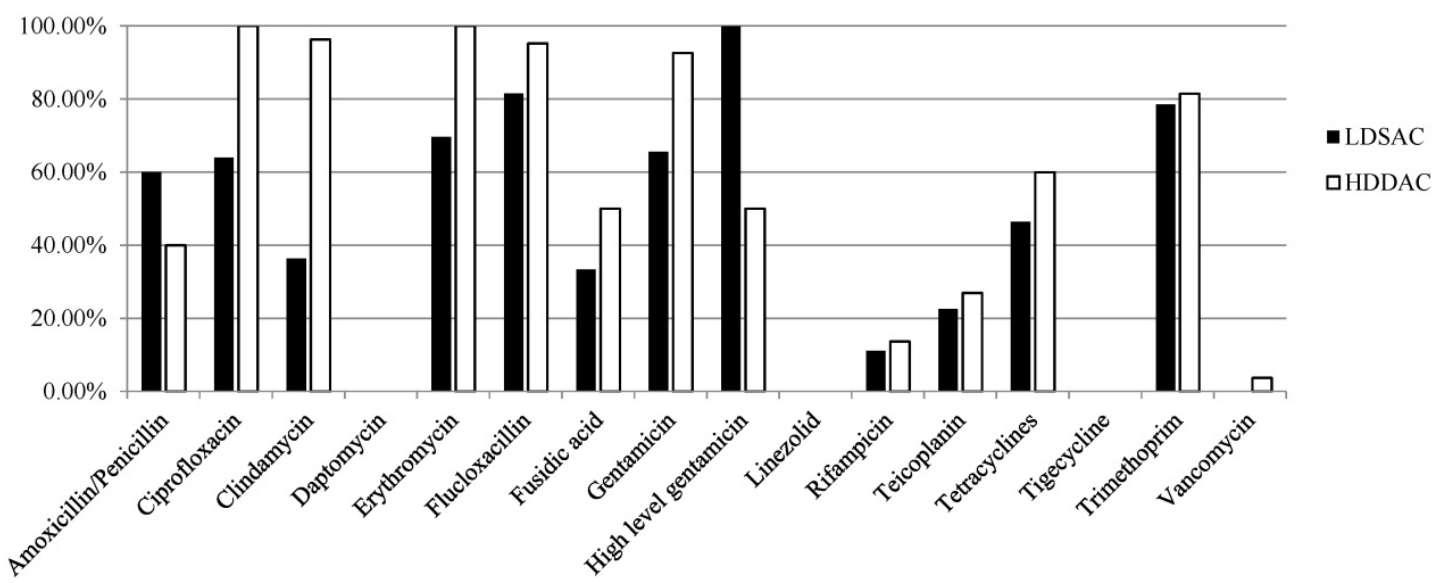

Figure 2. Resistance in Gram positive organisms

Table 2. Resistance rates in Gram positive organisms

\begin{tabular}{|c|c|c|c|c|c|c|c|}
\hline Antibiotic & $\begin{array}{l}\text { Number of } \\
\text { LDSAC } \\
\text { organisms tested }\end{array}$ & $\begin{array}{l}\text { Number of LDSAC } \\
\text { organisms resistant }\end{array}$ & $\begin{array}{l}\text { Percentage of } \\
\text { LDSAC organisms } \\
\text { resistant }(\%)\end{array}$ & $\begin{array}{l}\text { Number of } \\
\text { HDDAC } \\
\text { organisms tested }\end{array}$ & $\begin{array}{l}\text { Number of } \\
\text { HDDAC organisms } \\
\text { resistant }\end{array}$ & $\begin{array}{l}\text { Percentage of } \\
\text { HDDAC organisms } \\
\text { resistant (\%) }\end{array}$ & $\begin{array}{l}\text { Chi squared } \\
\text { test, p value }=\end{array}$ \\
\hline Amoxicillin & 5 & 3 & 60.00 & 5 & 2 & 40.00 & 0.564 \\
\hline Ciprofloxacin & 25 & 16 & 64.00 & 22 & 22 & 100.00 & $0.035^{*}$ \\
\hline Clindamycin & 33 & 12 & 36.36 & 27 & 26 & 96.30 & $0.000^{* *}$ \\
\hline Daptomycin & 11 & 0 & 0.00 & 18 & 0 & 0.00 & \\
\hline Erythromycin & 33 & 23 & 69.70 & 26 & 26 & 100.00 & 0.064 \\
\hline Flucloxacillin & 27 & 22 & 81.48 & 21 & 20 & 95.24 & 0.485 \\
\hline Fusidic acid & 27 & 9 & 33.33 & 22 & 11 & 50.00 & 0.176 \\
\hline Gentamicin & 32 & 21 & 65.63 & 27 & 25 & 92.59 & 0.084 \\
\hline High level gentamicin & 5 & 5 & 100.00 & 4 & 2 & 50.00 & 0.317 \\
\hline Linezolid & 30 & 0 & 0.00 & 23 & 0 & 0.00 & \\
\hline Rifampicin & 27 & 3 & 11.11 & 22 & 3 & 13.64 & 0.722 \\
\hline Teicoplanin & 31 & 7 & 22.58 & 26 & 7 & 26.92 & 0.641 \\
\hline Tetracycline & 28 & 13 & 46.43 & 25 & 15 & 60.00 & 0.319 \\
\hline Tigecycline & 13 & 0 & 0.00 & 18 & 0 & 0.00 & \\
\hline Trimethoprim & 28 & 22 & 78.57 & 27 & 22 & 81.48 & 0.865 \\
\hline Vancomycin & 32 & 0 & 0.00 & 27 & 1 & 3.70 & \\
\hline
\end{tabular}

Table 3. Resistance rates in Gram negative organisms

\begin{tabular}{|c|c|c|c|c|c|c|c|}
\hline Antibiotic & $\begin{array}{l}\text { Number of } \\
\text { LDSAC organisms } \\
\text { tested }\end{array}$ & $\begin{array}{l}\text { Number of LDSAC } \\
\text { organisms resistant }\end{array}$ & $\begin{array}{l}\text { Percentage of LDSAC } \\
\text { organisms resistant } \\
(\%)\end{array}$ & $\begin{array}{l}\text { Number of } \\
\text { HDDAC } \\
\text { organisms tested }\end{array}$ & $\begin{array}{l}\text { Number of } \\
\text { HDDAC organisms } \\
\text { resistant }\end{array}$ & $\begin{array}{l}\text { Percentage of } \\
\text { HDDAC organisms } \\
\text { resistant (\%) }\end{array}$ & $\begin{array}{l}\text { Chi squared } \\
\text { test, } p \text { value }=\end{array}$ \\
\hline Amoxicillin & 11 & 7 & 66.64 & 6 & 5 & 83.33 & 0.545 \\
\hline Aztreonam & 7 & 1 & 14.29 & 1 & 0 & 0.00 & 0.705 \\
\hline Ceftazidime & 10 & 3 & 30.00 & 6 & 1 & 16.67 & 0.551 \\
\hline Cefuroxime & 6 & 2 & 33.33 & 5 & 3 & 60.00 & 0.302 \\
\hline Ciprofloxacin & 13 & 2 & 15.38 & 8 & 1 & 12.50 & 0.835 \\
\hline Co-amoxiclav & 10 & 3 & 30.00 & 4 & 2 & 50.00 & 0.465 \\
\hline Ertapenem & 9 & 0 & 0.00 & 5 & 1 & 20.00 & \\
\hline Gentamicin & 14 & 5 & 35.71 & 8 & 1 & 12.50 & 0.272 \\
\hline Meropenem & 13 & 0 & 0.00 & 8 & 0 & 0.00 & \\
\hline Piperacilllin-Tazobactam & 12 & 0 & 0.00 & 7 & 1 & 14.29 & \\
\hline Tigecycline & 8 & 3 & 37.50 & 6 & 3 & 50.00 & 0.617 \\
\hline Trimethoprim & 12 & 6 & 50.00 & 6 & 2 & 33.33 & 0.564 \\
\hline
\end{tabular}

In Gram negative organisms, no significant change in resistance was observed (Table 3, Figure 3). No resistance to meropenem was observed in organisms isolated in either group.

Taking these organisms in the context of their infections, we considered the number of infections resistant to either clindamycin, gentamicin, or both. A polymicrobial infection was considered resistant if it contained at least one organism which demonstrated resistance to clindamycin or gentamicin. We assumed resistance to clindamycin in Gram negative bacteria and resistance to gentamicin in all enterococci. Despite the higher rate of resistance in infections that occur on HDDAC, there were still proportionally 


\section{Percentage of Resistant Gram negative Organisms}

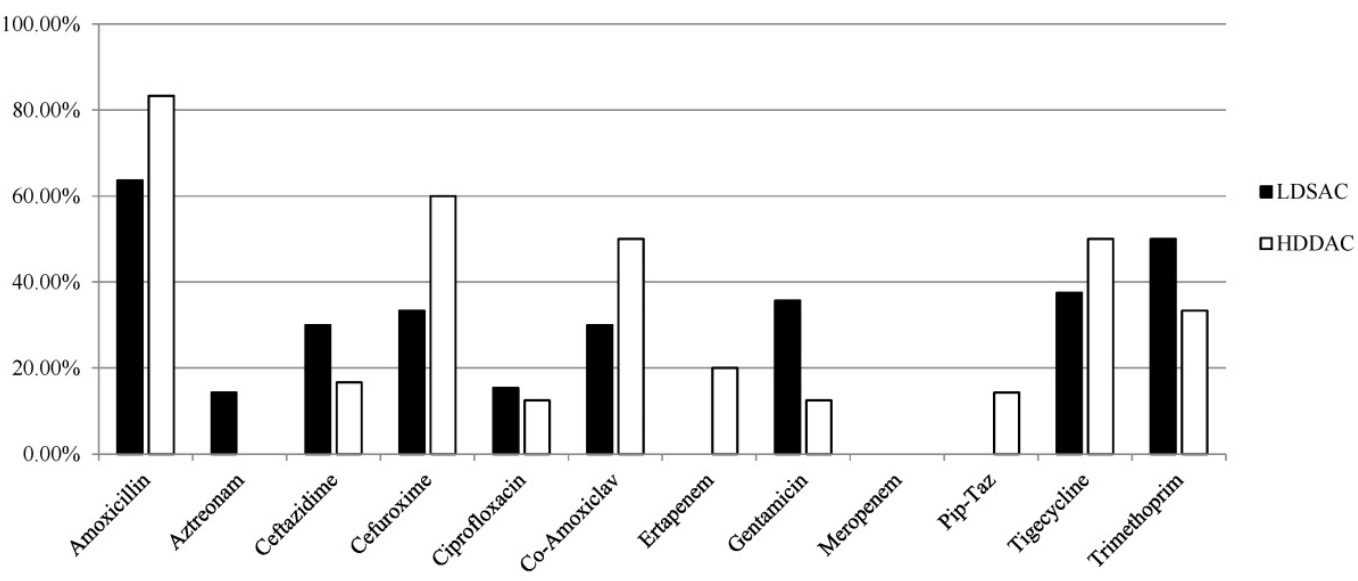

Figure 3. Resistance in Gram negative organisms

fewer infections resistant to clindamycin, gentamicin and to both antibiotics on HDDAC compared to LDSAC (Table 4). This is due to the significantly reduced overall infection rate on HDDAC. However, these differences were not found to be statistically significant.

Table 4. Number of resistant infections

\begin{tabular}{|c|c|c|c|}
\hline & $\begin{array}{l}\text { Low dose single } \\
\text { antibiotic cement }\end{array}$ & $\begin{array}{l}\text { High dose dual } \\
\text { antibiotic cement }\end{array}$ & $\begin{array}{l}\text { Chi squared } \\
\text { test, } \mathrm{p}=\end{array}$ \\
\hline 1941 hemiarthroplasties & 681 & 1260 & \\
\hline Deep SSI & 23 (3.4\%) & 15 (1.2\%) & $0.003^{* *}$ \\
\hline $\begin{array}{l}\text { Deep SSI resistant to } \\
\text { clindamycin }\end{array}$ & $14(2.06 \%)$ & 15 (1.19\%) & 0.134 \\
\hline $\begin{array}{l}\text { Deep SSI resistant to } \\
\text { gentamicin }\end{array}$ & $10(\mathbf{1 . 4 7 \% )}$ & $12(1.00 \%)$ & 0.305 \\
\hline $\begin{array}{l}\text { Deep SSI resistant to } \\
\text { both }\end{array}$ & $8(1.17 \%)$ & $12(\mathbf{1 . 0 0} \%)$ & 0.643 \\
\hline
\end{tabular}

\section{Discussion}

Gentamicin loaded bone cement has been shown to be an effective prophylactic measure against SSI in arthroplasty patients. ${ }^{12}$ With double the dose of gentamicin and with the addition of clindamycin, it has been shown in vitro that HDDAC inhibits bacterial growth for longer when compared to LDSAC. ${ }^{8}$ Kendall et al. experimented with the addition of tobramycin and vancomycin to gentamicin-loaded cement discs in vitro. They found antibacterial growth was inhibited further, but that it was not completely eradicated. ${ }^{9}$ Other concerns have been raised about the development of resistance in antibiotic loaded bone cement. ${ }^{10}$ Release of sub-inhibitory concentrations of antibiotics has been suggested as a possible mechanism for this, by conferring adaptational mutations in bacteria.9,13 However, in contrast, a recently published paper compared antibiotic resistance in PJI organisms in primary arthroplasty and found no major differences with the introduction of antibiotic-loaded bone cement. ${ }^{14}$ In general, there exists very little clinical research in the literature on antibiotic resistance profiles in the context of antibiotic loaded bone cement and it still remains a topic of debate. This was the stimulus for our study.

An analysis of 125 acute care hospitals in England demonstrated a cumulative SSI incidence rate of $4.06 \%$ in hip hemiarthroplasty patients ${ }^{5}$ The infection rates found in our study (3.4\% for LDSAC and $1.2 \%$ for HDDAC) are comparable to this and to the findings of the quasi-randomised controlled trial within our trust. The trial demonstrated an infection rate of $3.5 \%$ in LDSAC and $1.1 \%$ in HDDAC. These similarities are to be expected since, although the data sets are not the same, they do include many of the same patients (Figure 1).

The 2013/14 Public Health Report on SSI in England reported that $27 \%$ of neck of femur SSIs were polymicrobial in nature. ${ }^{15}$ However, in our study we found $52 \%$ of infections to be polymicrobial in LDSAC and $53 \%$ in HDDAC. The reason for this difference is not clear but could represent improved culturing methods, or a genuinely higher rate of polymicrobial infections.

Nationally, S. aureus (including MRSA) was found to account for $26 \%$ of neck of femur SSIs. ${ }^{15}$ In our study, it accounted for 19\% of infections in LDSAC, yet was eradicated with the use of HDDAC. This may be explained by the introduction of a MSSA decolonisation programme to the units in September 2011. In our results, no Staphylococcus aureus organisms were isolated from the infections between the introduction of this programme in September 2011 and the introduction of HDDAC for all our hemiarthroplasty patients in May 2012. There were only three infections in this window, (these were all polymicrobial infections in LDSAC patients) so the 
results are biased by very low numbers, but this may in part represent the efficacy of our S. aureus decolonisation methods. It may also be speculated that the significantly higher levels of gentamicin and clindamycin in the HDDAC cemented hips may have eradicated $S$. aureus, and many coagulase negative staphylococci, due to the synergistic release and antimicrobial efficacy properties of both antibiotics.

We would expect local antibiotics to treat only susceptible organisms. Therefore, it is understandable that highly resistant pathogens may not be eradicated despite the high concentrations in situ. In the cases infected with Gram positive organisms, we found a proportionally higher level of resistance among the surviving pathogens- most notably to clindamycin between LDSAC and HDDAC. This finding may be partly explained by the higher number of $S$. epidermidis bacteria among the deep infections in the HDDAC group. The presence of gentamicin and clindamycin may prevent infection caused by more sensitive bacteria, allowing more resistant but lower virulence organisms such as $S$. epidermidis to become relatively more common in the HDDAC cohort overall, albeit with fewer total infections, and fewer (statistically similar) resistant infections. Resistance in this group was also proportionally higher to ciprofloxacin, with all staphylococci found to be resistant to this antibiotic. We are unsure as to the mechanism of this resistance. This finding may just be biased by low numbers and not represent a true association. Ciprofloxacin resistance is not uncommon among the coagulase negative staphylococci and this finding may just have occurred by chance. Otherwise it may, in part, represent the higher number of $S$. epidermidis infections in the HDDAC group ( $S$. epidermidis is frequently more resistant than other organisms known to cause hip hemiarthroplasty infections in our organisation). Increases in resistance were also seen to several other antibiotics, though these were not statistically significant. In the group of Gram negative bacteria the changes in resistance levels were generally low.

Despite the observed variations in the type of infecting organisms and resistance profiles against some antibiotics among particularly Gram positive pathogens, it was an important finding that the levels of resistance to those antibiotics used for treatment of PJI remained largely unchanged (e.g. rifampicin, daptomycin, vancomycin or tigecycline against Gram positive, and ciprofloxacin, meropenem or ceftazidime against Gram negatives). It is therefore reassuring to know that these antimicrobial options remain effective and do not lower the probability for a successful eradication of the infection.
Further to this, with the use of HDDAC we found that, such is the reduced infection rate, there was also a trend to a lower rate of resistance when compared to LDSAC (Table 4). Although these differences were not found to be statistically significant, it is reassuring for teams using HDDAC to prevent SSI in hip hemiarthroplasty.

Antibiotic resistance has long been a concern with the use of antibiotic-loaded bone cement. ${ }^{9}$ However, to our knowledge, this study represents the first clinical data comparing antibiotic resistance profiles between high dose dual antibiotic cement and low dose single antibiotic cement in hip hemiarthroplasty.

With 24,000 patients undergoing hip hemiarthroplasty in the UK annually ${ }^{2}$ we can extrapolate our results to this cohort. This would generate 816 infections if LDSAC was used and 288 infections with HDDAC. Of these, resistance to both clindamycin and gentamicin would be seen in 282 patients with LDSAC and 229 patients with HDDAC. These figures are shown in Table 5. In this study, we have demonstrated that HDDAC is not driving antibiotic resistant SSI in the hip hemiarthroplasty population and would advocate its continued use in this high-risk subset of patients.

Table 5. Extrapolation of our results to a national cohort

\begin{tabular}{lll}
\hline NHS as a whole (extrapolated) & LDSAC & HDDAC \\
\hline 24,000 hemiarthroplasties & 24,000 & 24,000 \\
Deep SSI & 816 & 288 \\
Deep SSI resistant to clindamycin & 493 & 288 \\
Deep SSI resistant to gentamicin & 352 & 229 \\
Deep SSI resistant to both & 282 & 229
\end{tabular}

High dose dual antibiotic cement $=$ Copal G+C, Heraeus Medical, UK

Low dose single antibiotic cement $=$ Palacos R+G, Heraeus Medical, UK

\section{Limitations}

Unfortunately, detailed study of systemic antibiotic use in each individual patient prior to acquiring SSI was beyond the scope of this study. All patients in this study received systemic prophylactic antibiotics according to our trust protocol for hip hemiarthroplasty.

\section{Competing Interests}

Our senior author, Mike Reed, and co-author Martin Marsh, are speakers for Heraeus. There are no conflicts to declare for all other authors.

\section{References}

1. Gullberg B, Johnell O, Kanis J. World-wide Projections for Hip Fracture. Osteoporosis International. 1997; 7(5): 407-413.

2. National Hip Fracture Database. Available online.

3. Jameson S, Khan, Reed $\mathrm{M}$ et al. A national analysis of complications following hemiarthroplasty for hip fracture in older patients. QJM. 2012; 105(5): 455-460. 
4. Coello R, Charlett A, Borriello P et al. Adverse impact of surgical site infections in English hospitals. Journal of Hospital Infection. 2005; 60(2): 93-103.

5. Wilson J, Charlett A, Duckworth G et al. Rates Of Surgical Site Infection After Hip Replacement As A Hospital Performance Indicator: Analysis Of Data From The English Mandatory Surveillance System. Infection Control and Hospital Epidemiology. 2008 Mar; 29(3): 219-226. Web.

6. Sprowson A, Jensen C, Reed M, et al. The use of high-dose dual-impregnated antibiotic-laden cement with hemiarthroplasty for the treatment of a fracture of the hip. Bone Joint Journal. 2016; 98B: 1534-1541.

7. Gehrke T, Forster G, Frommelt L. Pharmacokinetic Study of a Gentamicin/Clindamicin Bone Cement Used in One-stage Revision Arthroplasty. Bone Cements and Cementing Technique, 2001: 127-134.

8. Ensing G, van Horn J, Neut D et al. Copal Bone Cement Is More Effective in Preventing Biofilm Formation than Palacos R-G. Clinical Orthopaedics and Related Research. 2008 Jun; 466(6): 1492-1498.

9. Kendall RW, Duncan CP, Ngui-Yen JH et al. Persistence of bacteria on antibiotic loaded acrylic deposits: a reason for caution. Clinical Orthopaedics and Related Research. 1996 Aug; 329: 273-280

10. Thornes B, Murray P, Bouchier-Hayes D. Development of resistant strains of Staphylococcus epidermidis on gentamicin-loaded bone cement in vivo. The Journal of Bone and Joint Surgery. 2002 Jul; 84(5): 758-760.

11. Public Health England. Investigation of orthopaedic implant associated infections. UK Standards for Microbiology Investigations. 2016; B44: Issue 2

12. Chang $Y$, Tai $C$, Ueng $S$ et al. Gentamicin in bone cement: A potentially more effective prophylactic measure of infection in joint arthroplasty. Bone and Joint Research. 2013; 2(10): 220-226.

13. Neut $D$, van de Belt $H$, Busscher $H$ et al. Residual gentamicin-release from antibiotic-loaded polymethylmethacrylate beads after 5 years of implantation. Biomaterials. 2003; 24(10): 1829-1831.

14. Hansen EN Adeli B, Parvizi J et al. Routine use of antibiotic laden bone cement for primary total knee arthroplasty: impact on infecting microbial patterns and resistance profiles. J Arthroplasty. 2014; 29(6): 1123-1127

15. Public Health England. Surveillance of surgical site infections in NHS hospitals in England, 2013/14. Available online. 